\title{
Thermodynamic Consistency Lines of the 511 Mutual Solubility Data and the VLE Data of 7262 Constant-Temperature and 5167 Constant-Pressure Binaries
}

\author{
Satoru KATO* ${ }^{*}$ and Hiroshi TACHIBANA \\ Department of Applied Chemistry, Tokyo Metropolitan University \\ 1 -1 Minamiohsawa, Hachiohji, Tokyo 192-0397, Japan \\ (Received January 2, 2011; Accepted February 22, 2011)
}

\begin{abstract}
It has already been shown that the binary vapor-liquid equilibrium (VLE) data form thermodynamic consistency (TC) lines, defined using the polarity exclusion factor; i.e., the ratio of data deviation from the Gibbs-Duhem equation, $F$, to $|B-A|$, where $A$ and $B$ denote Margules parameters representing the VLE data. The present investigation shows that the mutual solubility data from the literature converge to TC lines. It was shown that fluctuations of the TC lines from the LLE data are less than those from the VLE data.
\end{abstract}

\section{Introduction}

Mutual solubility data are of practical importance for the rational design of solvent selection and the prediction of ternary liquid-liquid equilibria (LLE). A significant number of mutual solubility data can be found in the literature [1]. However, a method for determining the thermodynamic consistency of the mutual solubility data has not yet been established. Meanwhile, Kato [2] recently showed that TC lines are useful for the thermodynamic consistency (TC) identification of the binary vapor-liquid equilibrium (VLE) data [3]; i.e., it was shown that each binary has a straight TC line represented as $\beta=a P^{b}$, where $\beta$ and $P$ denote the polarity exclusion factor and the system pressure, respectively, while $a$ and $b$ are constants. It has already been shown that the TC line for the 1-butanol(1)-water(2) binary determined from the VLE data is identical with that determined from the binary LLE data. It was also suggested that the data convergence of the TC line from the LLE data are much better than that from the VLE data [2].

The purpose of the present investigation is to show that all of the mutual solubility data from the literature conform to their TC lines, and that their data convergences are excellent.

\section{Modeling}

2.1 Determination of Margules parameters, $A$ and $B$, representing the data

Liquid-liquid equilibria for components 1 and 2 at temperature $T$ are given as follows:

$$
\begin{aligned}
& \left(\gamma_{1} x_{1}\right)_{1}=\left(\gamma_{1} x_{1}\right)_{2} \\
& \left(\gamma_{2} x_{2}\right)_{1}=\left(\gamma_{2} x_{2}\right)_{2}
\end{aligned}
$$

where $\gamma_{i}$ and $x_{i}$, respectively, denote the activity coefficient of component $i$ and the mole fraction of component $i$, while ( $)_{j}$ denotes the value in phase $j$. As shown in the literature [1], the UNIQUAC equation can represent all of the mutual solubility data in the literature; therefore, the present investigation uses the UNIQUAC equation for formulating $\gamma_{i}$ as follows: 


$$
\begin{aligned}
& \ln \gamma_{i}=\ln \frac{\Phi_{i}}{x_{i}}+\frac{z}{2} q_{i} \ln \frac{\theta_{i}}{\Phi_{i}}+\Phi_{i}\left(l_{i}-\frac{r_{i}}{r_{j}} l_{j}\right)-q_{i} \ln \left(\theta_{i}+\theta_{j} \tau_{j i}\right) \\
& +\theta_{j} q_{i}\left(\frac{\tau_{i j}}{\theta_{i}+\theta_{j} \tau_{j i}}-\frac{\tau_{i j}}{\theta_{j}+\theta_{i} \tau_{i j}}\right) \\
& \Phi_{i}=\frac{x_{i} r_{i}}{x_{1} r_{1}+x_{2} r_{2}} \\
& \theta_{i}=\frac{x_{i} q_{i}}{x_{1} q_{1}+x_{2} q_{2}} \\
& l_{i}=\frac{z}{2}\left(r_{i}-q_{i}\right)-\left(r_{i}-1\right)
\end{aligned}
$$

where $r_{i}$ and $q_{i}$ denote the molar volume and molecular surface area of component $i$, respectively. In Eq. (3), $z$ denotes the coordination number. In the following analysis, $z=10$ was used. From Eqs. (1) to (3), the interaction parameters, $\tau_{12}$ and $\tau_{21}$, can be determined, if the mutual solubilities, $\left(x_{1}\right)_{1}$ and $\left(x_{1}\right)_{2}$, are known. From the $\tau_{12}$ and $\tau_{21}$ values, the infinite dilution activity coefficients, $\gamma_{1}^{\infty}$ and $\gamma_{2}^{\infty}$, are determined from Eq. (3) at $x_{1}=0$ and $x_{2}=0$, respectively. Using $\gamma_{1}^{\infty}$ and $\gamma_{2}^{\infty}$, the Margules parameters, $A$ and $B$, are determined as follows:

$$
\begin{aligned}
& A=\ln \gamma_{1}^{\infty} \\
& B=\ln \gamma_{2}{ }^{\infty}
\end{aligned}
$$

Therefore, $A$ and $B$ can be fixed, if the mutual solubility, $\left(x_{1}\right)_{1}$ and $\left(x_{1}\right)_{2}$, are known.

Meanwhile, the VLE values for components 1 and 2 are given as follows:

$$
\begin{aligned}
& P y_{1}=\gamma_{1} x_{1} p_{1 s} \\
& P y_{2}=\gamma_{2} x_{2} p_{2 s}
\end{aligned}
$$

where $P, y_{i}$ and $p_{i s}$, respectively, denote the system pressure, the mole fraction of component $i$ in the vapor phase and the vapor pressure of component $i$. As shown in the literature [3], the VLE data can be satisfactorily correlated by the Margules equation; therefore, the present investigation uses the Margules equation as follows:

$$
\begin{aligned}
& \ln \gamma_{1}=x_{2}{ }^{2}\left[A+2(B-A) x_{1}\right] \\
& \ln \gamma_{2}=x_{1}{ }^{2}\left[B+2(A-B) x_{2}\right]
\end{aligned}
$$

The $A$ and $B$ values represent the constant-temperature $P-x$ data and constant-pressure $T-x$ data.

\subsection{Determination of the TC lines}

At constant temperature and pressure, the activity coefficients of a binary system must satisfy the following Gibbs-Duhem (GD) equation [4]:

$$
\begin{aligned}
& D=0 \\
& D=x_{1} \frac{d \ln \gamma_{1}}{d x_{1}}+\left(1-x_{1}\right) \frac{d \ln \gamma_{2}}{d x_{1}}
\end{aligned}
$$


where $D$ denotes the deviation from the GD equation, i.e., Eq. (10). In a previous investigation [5], $D$ was approximated using a finite difference method, and it was shown that $\mathrm{D}$ is simply formulated as follows:

$$
D=2(B-A) \Delta x_{1}{ }^{2}
$$

where $\Delta x_{1}$ denotes the experimental error involved in $x_{1}$. Equation (12) clearly shows that $D=0$ if $A=B$ holds in spite of the significant notable experimental error represented by $\Delta x_{1} \neq 0$. Meanwhile, $D=0$ is also satisfied at $\Delta x_{1}=0$, even if $\mathrm{A} \neq \mathrm{B}$, as is the case in highly polar mixtures. Based on these facts, the following polarity exclusion factor, $\beta$, was defined [2], and an empirical relationship was proposed as follows:

$$
\beta \equiv \frac{F}{|B-A|}=a P^{b}
$$

where $a$ and $b$ are constants, and $P$ denotes the system pressure. If constant-temperature data are examined, $P$ is replaced by the average vapor pressure, $p_{\mathrm{s} \text {,ave }}$, as follows:

$$
P=\frac{p_{1 s}+p_{2 s}}{2} \equiv p_{s, a v e}
$$

Using the VLE data including 7262 constant-temperature and 5167 constant-pressure binaries, it was shown that Eq. (13) holds at each binary [2]. In Eq. (13), $F$ represents the VLE data deviation from $A=B$ as follows:

$$
F=\frac{100}{n} \sum_{k=1}^{n}\left|\frac{y_{1 k, \text { smooth }}-y_{1 k, M 1}}{y_{1 k, M 1}}\right|
$$

where $y_{1 k, \text { smooth }}$ denotes the vapor phase mole fraction of component 1 , calculated using the $A$ and $B$ values representing the $P-x$ and $T-x$ data. In Eq. (15), $y_{1 k, M 1}$ denotes the vapor phase mole fraction of component 1, calculated using the following one binary parameter Margules equation:

$$
\begin{aligned}
& \ln \gamma_{1}=x_{2}{ }^{2} E \\
& \ln \gamma_{2}=x_{1}{ }^{2} E
\end{aligned}
$$

where $E$ denotes the one-binary parameter. The $E$ value is determined at each $x_{1}$ point using the following restriction:

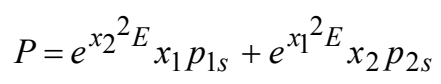

where $P$ is the smoothed pressure calculated using the $A$ and $B$ values representing the $P-x$ and $T-x$ data. In the following analysis, the $y_{1 k, \text { smooth }}$ values at $k=41$ for different-compositions with an equal increment in $x_{1}$, ranging from $0 \leqq x_{1} \leqq 1$, were used. It should be noted that on the GD equation, $D=0$ is satisfied, if $F=0$, because, in that case, the VLE data satisfy $A=B$, which holds in the case of simple liquid mixtures and non-polar binaries. Therefore, $F$ is a measure of the data deviation from simple liquid mixtures, and hence, it represents the mixture polarity strength, as also does $|B-A|$.

The TC line, Eq. (13), is useful for the prediction of not only the mutual solubility, but also the ternary LLE; therefore, in the following section, the TC line is applied to the LLE data including 96 constant-temperature binaries; , i.e., mutual solubility data.

\section{Data Sources}

The mutual solubility data compiled in the Dechema Chemistry Data Series [1] were used in the following analysis. The 96 binaries including water as one of the components were chosen, because their vapor pressures are found in ref. [3]. The $F$ and $\beta$ values were calculated for 511 points at different temperatures. The VLE data compiled in ref. [3] were also used, and their $F$ and $\beta$ values were compared with those from the LLE data. Table 1 lists the binaries classified into chemical groups given in the 
Dechema Chemistry Data Series [3]. Table 1 includes the numbers of the constant-temperature data sets, $n_{T}$, and constant-pressure data sets, $n_{P}$, for each group. Table 1 also includes the average values of $|B-A|$, $|B-A|_{\text {ave }}$, and the average values of $F, F_{\text {ave }}$.

Table 1 Classification of the VLE and LLE data

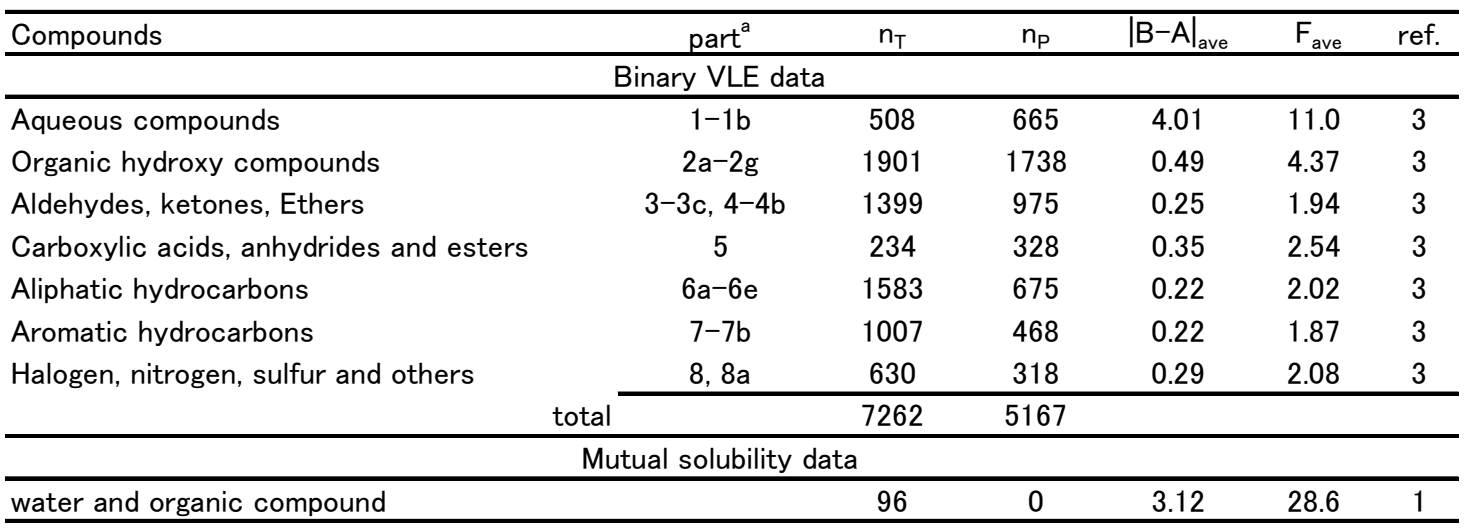

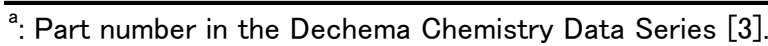

\section{Results and Discussion}

\section{1 Effect of polarity on $F$ and $|B-A|$}

Using the 12429 VLE data sets, the controlling factor of $F$ is examined. In Fig. 1, as an example, $y_{1}$ calculated using the $A$ and $B$ values, $y_{1, \text { smooth }}$, is plotted versus $x_{1}$ for the methanol(1)-water(2) binary at $333.15 \mathrm{~K}$. The $A$ and $B$ values were taken from ref. [3], because the Dechema Chemistry Data Series [3] compile Margules binary parameters, $A$ and $B$, representing the $P-x$ and $T-x$ data. Figure 1 includes the $y_{1, \mathrm{M} 1}$ values calculated using the one binary parameter Margules equation, Eqs. (16) to (18). Using the two lines in Fig. 1, the $F$ value of the data set can be determined from Eq. (15). In Fig. 2, cumulative polygons are plotted versus $F$ using the VLE data sets listed in Table 1. The total number, $n_{\text {total }}=n_{T}+n_{P}$, is listed in Table 1 for each chemical group. Figure 2 demonstrates that the polarity is a controlling factor of $F$, because the $F$ values of aqueous systems are much higher than those of hydrocarbons. As shown in Table 1, these binaries consisting of polar compounds have high values of $|B-A|$ and $F$.

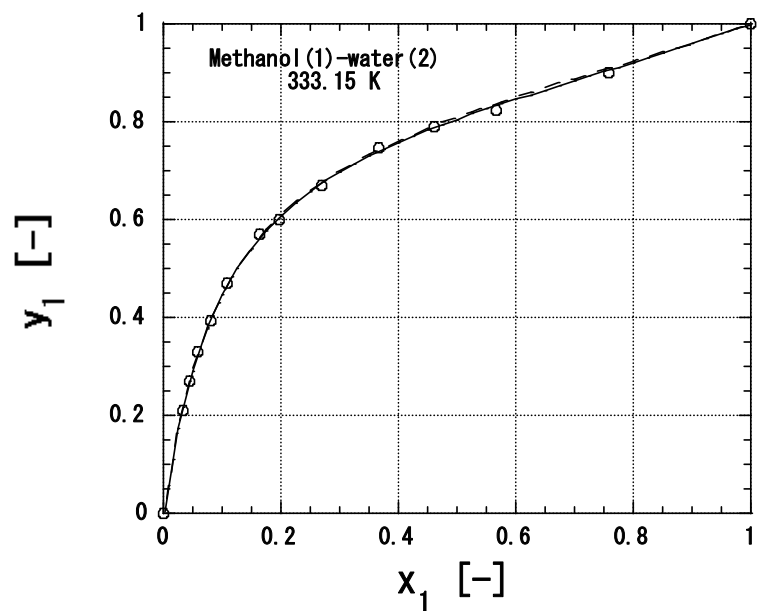

Figure 1. Relationships between $x_{1}$ and $y_{1}$ for the methanol(1)-water(2) binary at $333.15 \mathrm{~K}$, (०) $x-y$ data from ref. [3], (-) $y_{1, \text { smooth }}$ calculated using $A=0.6916$ and $B=0.5626$ compiled in ref. [3], (- - -) $y_{1, M 1}$ calculated from the one binary parameter Margules equation, Eqs. (16) to (18).

In Figs. 3(a) and (b), mutual solubility data are plotted, in which the $(\times)$ and $(\bullet)$ symbols, respectively, denote the original data from ref. [1] and smoothed data compiled in ref. [1]. Using the smoothed data, $A$ and $B$ values were determined from Eqs. (1) to (5) at different temperatures. Using the $A$ and $B$ values, in 
Fig. 4, $y_{1, \text { smooth }}$ and $y_{1, \mathrm{M} 1}$ are plotted versus $x_{1}$ for the 2-butanone(1)-water(2) binary at $273.15 \mathrm{~K}$. Although the solutions between the mutual solubility values, $\left(x_{1}\right)_{2}<x_{1}<\left(x_{1}\right)_{1}$, are hypothetical, continuous $y_{1, \text { smooth }}$ and $y_{1, M 1}$ functions exist, as shown in Fig. 4. Therefore, the $F$ values can be calculated using Eq. (15) for the mutual solubility data. One of the advantages of the application of Eq. (15) to hypothetical solutions is that, as has already been shown for the 1-butanol(1)-water(2) binary [2], the mutual solubility data provide the same TC line with that determined from the constant-temperature and constant-pressure VLE data. In Fig. 5 using the $\bigcirc$ symbols, a cumulative polygon is plotted versus $F$ for the 96 LLE binaries including water. Figure 5 includes the cumulative polygon for the 1073 VLE data sets including water, as denoted by the solid line. Figure 5 shows that the polarity of the two-liquid phase forming LLE binaries is higher than that for the aqueous VLE data. In Table 1, the average values of $F$ and $|B-A|$ for the mutual solubility data are listed. Table 1 shows that $F_{\text {ave }}$ of the LLE data is higher than that for the VLE data.

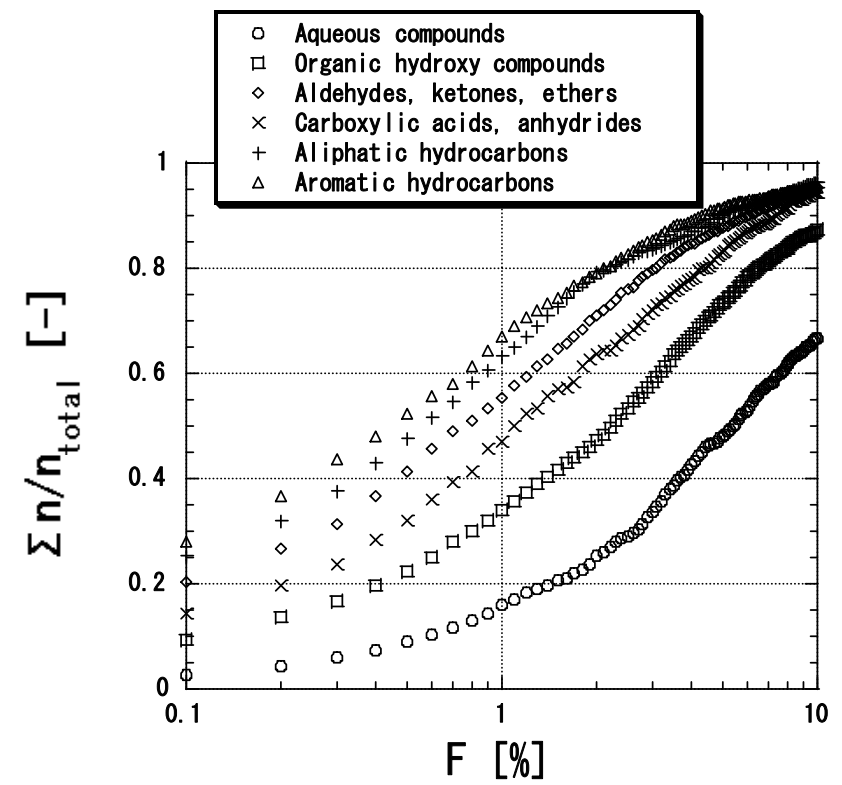

Figure 2. Relationships between the deviation from $A=B$ and the ratio of the cumulative number of data sets to the total number of the chemical groups from the VLE data.
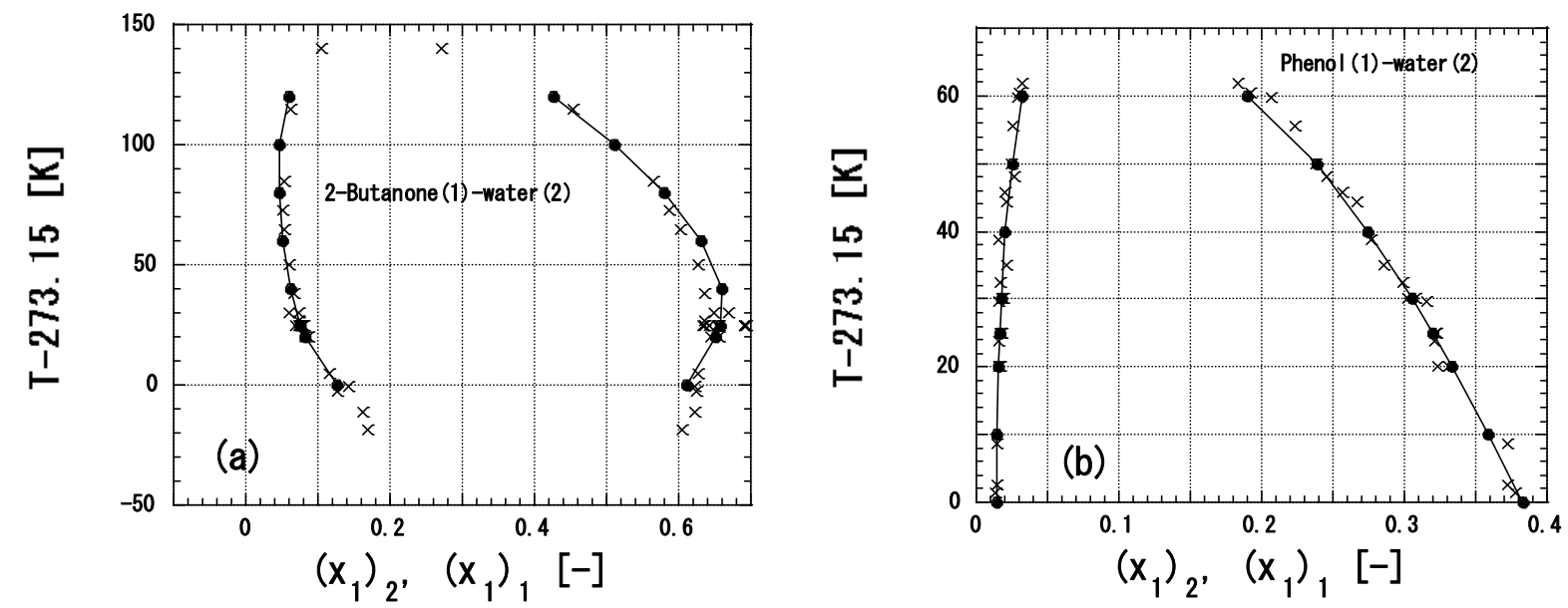

Figure 3. Mutual solubility data of the (a) 2-butanone(1)-water(2) and (b) phenol(1)-water(2) binaries, $(\times)$ original data [1], $(\bullet)$ smoothed data cited from Dechema Data Series[1], (-) connecting line for the smoothed data. 


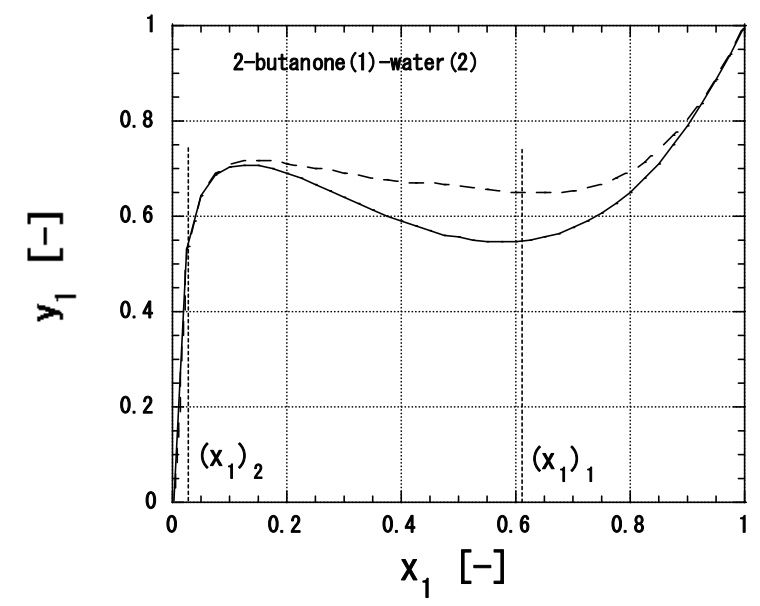

Figure 4. Relationships between $x_{1}$ and $y_{1}$ for the 2-butanone(1)-water(2) binary at $273.15 \mathrm{~K}$, (-) $y_{1, \text { smooth }}$ and (- - ) $y_{1, M 1}$, where $A=\ln \gamma_{1}^{\infty}=3.030$ and $B=\ln \gamma_{2}^{\infty}=2.043$.

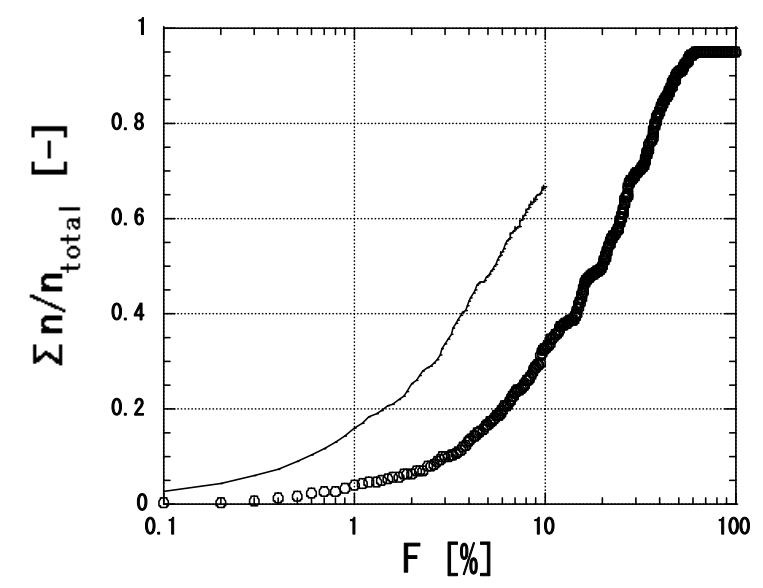

Figure 5. Relationships between the deviation from $A=B, F$, and the ratio of the cumulative number of data sets to the total number, $(\bigcirc)$ the 96 LLE binaries including water and $(-)$ the 1073 VLE data sets including water.

\subsection{Characteristics of the TC lines determined from the mutual solubility data}

In Fig. 6 , the polarity exclusion factor, $\beta$, is plotted versus $\left(p_{1 \mathrm{~s}}+p_{2 \mathrm{~s}}\right) / 2$ for the 2-butanone(1)-water(2) and phenol(1)-water(2) binaries. The $\times$ symbol at $p_{s, a v e}=400 \mathrm{kPa}$ in Fig. 6 corresponds to the mutual solubility data at $\mathrm{T}-273.15=140 \mathrm{~K}$ in Fig. 3a. Figure 6 shows that the mutual solubility data provide converged straight lines between $\ln \beta$ and $\ln \left[\left(p_{1 \mathrm{~s}}+p_{2 \mathrm{~s}}\right) / 2\right]$, i.e., TC lines. All of the mutual solubility data provided similar TC lines. To examine the data convergence of the TC lines, an average absolute deviation, $A A D$, is defined as follows

$$
A A D=\frac{100}{n} \sum_{i=1}^{n}\left|\frac{\beta_{i}-\beta_{i, T C}}{\beta_{i, T C}}\right|
$$

where $\beta_{T C}$ denotes the $\beta$ value calculated using the TC line. The average of the AAD values calculated using the smoothed mutual solubility data was as low as $1.8 \%$ for the 96 LLE binaries, which is lower than $3.1 \%$ determined from the VLE data consisting of the two-liquid phase forming binaries. Therefore, it can be concluded that, not only the VLE data, but also the LLE data form TC lines [2]. 


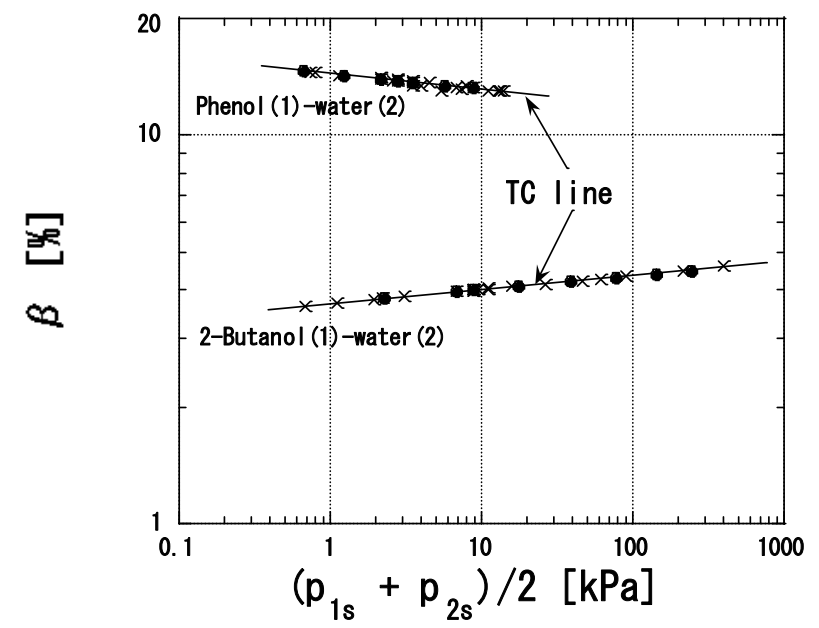

Figure 6. Relationships between $\beta$ and $\left(p_{1 \mathrm{~s}}+p_{2 \mathrm{~s}}\right) / 2$ for the 2-butanone(1)-water(2) and phenol(1)-water(2) binaries, $(\times)$ original data [1], $(\bullet)$ smoothed data cited from Dechema [1].

To examine the effect of polarity on $\beta$, in Fig. 7, a cumulative polygon is plotted versus $\beta$ for the LLE data using the $\bigcirc$ symbols. Figure 7 includes a cumulative polygon calculated for the VLE data including water, as denoted by the solid line. Figure 7 shows that, in spite of the significant differences in polarity appearing in Fig. 5 between the LLE and VLE data, the $\bigcirc$ symbols and solid line are almost overlapping. Therefore, the effect of the polarity is excluded from $\beta$. This is because the ratio of $F$ to $|B-A|$, as shown by Eq. (13), cancels out the effects of the polarity. Similar trends were found in the different chemical groups of the VLE data [2].

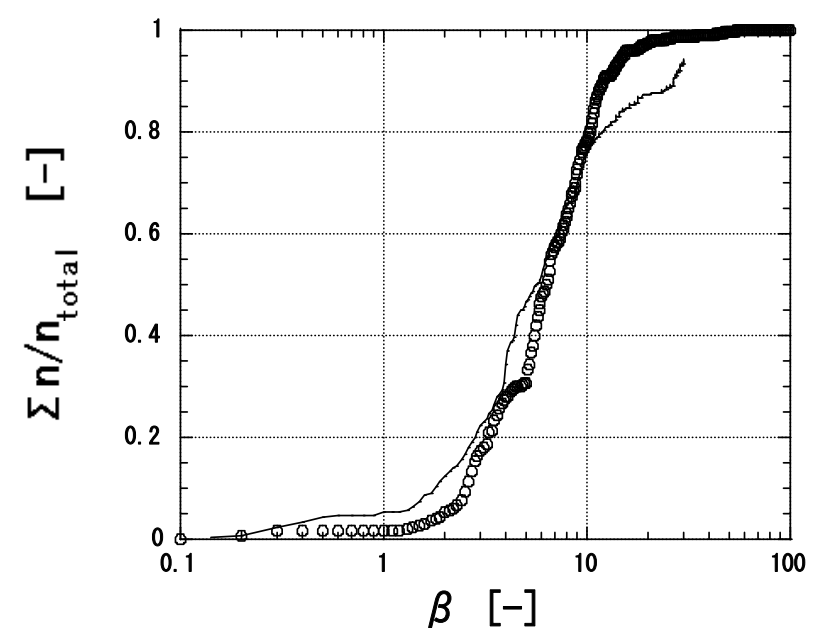

Figure 7. Relationships between $\beta$ and the ratio of the cumulative number of data sets to the total number, (O) the LLE data of the 96 binaries including water and (-) the 1073 VLE data sets including water.

To examine the data convergence of the TC lines determined from the LLE data, in Fig. 8, a cumulative polygon is plotted versus $100\left|\left(\beta-\beta_{T C}\right) / \beta_{T C}\right|$ for the smoothed mutual solubility data using the $\bigcirc$ symbols. Figure 8 also includes a cumulative polygon for the VLE data of the two-liquid phase forming binaries, as noted using the $\times$ symbols. Figure 8 shows that the fluctuations of the TC lines from the LLE data are less than those from the VLE data, because the $\bigcirc$ symbols are located above the $\times$ symbols. 


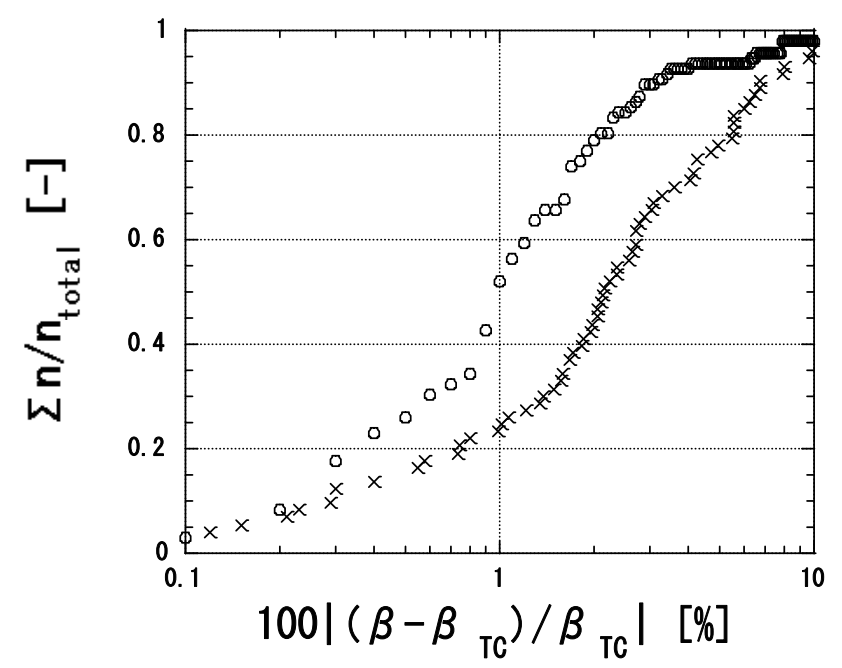

Figure 8. Relationships between $100\left|\left(\beta-\beta_{\mathrm{TC}}\right) / \beta_{\mathrm{TC}}\right|$ and the ratio of the cumulative number of data sets to the total number, $(\bigcirc)$ the LLE data of the 96 binaries including water and $(\times)$ the 73 VLE data sets including two-liquid phase forming binaries.

\section{Conclusion}

Using the mutual solubility data in the literature, the applicability of the TC lines to the LLE data was investigated. It was shown that the LLE data of the 96 binaries including water provide converged TC lines. The polarity of the two-phase forming LLE data is higher than that of the VLE data including water. However, the polarity does not have an effect on the polarity exclusion factor, $\beta=F /|B-A|$, as are the cases in the VLE data. The data convergences of TC lines from the LLE data are by no means inferior to those from the VLE data of the two-liquid phase forming binaries.

\section{References}

1) J.M. Sorensen, W. Arlt: Liquid-Liquid Equilibrium Data Collection, Binary Systems, Chemistry Data Series, Vol. V, Part 1, DECHEMA, (1979).

2) S. Kato, Fluid phase Equilibria, 302, 202 (2011).

3) J. Gmehling, U. Onken: Vapor-Liquid Equilibrium Data Collection, Dechema Chemistry Data Series, Vol. I, parts 1 to 8a, DECHEMA, (1977)-(2001).

4) J.M. Prausnitz: Molecular Thermodynamics of Fluid-Phase Equilibria, Prentice-Hall, New Jersey, (1969).

5) S. Kato, Fluid Phase Equilib. 297, 192 (2010). 